\title{
PERFORMANCE LIMITS FOR CELLULAR MULTIUSER COMMUNICATION SYSTEMS
}

Robert J. McEliece

Department of Electrical Engineering

California Institute of Technology

Pasadena, CA 91125

\section{Summary.}

In this paper we will define and study what we call "cellular" multiuser communication systems (MCSs). These systems can be thought of as models for cellular telephone systems using FDMA or TDMA multiaccess protocols.

A cellular MCS is characterized by a set of $N$ cells, and a set of $n$ channels. Within each cell, a certain number of two-way conversations ("calls") may take place, with each call occupying a distinct channel. In certain circumstances a given channel may be used simultaneously in several different cells. This permissible channel reuse is described by a collection of subsets of cells, called the "maximal reuse sets," the idea being that a given channel may be used simultaneously in each cell of any maximal reuse set.

We also characterize the demand for call services, by assuming that call requests arrive and depart randomly throughout the system according to a probabilistic model we leave largely unspecified, except to say that it is measured in Erlangs per channel, a parameter we shall call the offered traffic and denote by $r$. Thus if the offered traffic is $r$, the expected number of offered calls is $r n$. We introduce the "traffic pattern" $p=\left(p_{1}, p_{2}, \ldots, p_{n}\right)$, with $p_{i}$ representing the fraction of the total offered traffic present in the $i$ th cell. Thus if the offered traffic is $r$, the expected number of offered calls in the $i$ th cell is $p_{i} r n$. We assume that there is a channel assignment algorithm, which decides, for a given offered call, whether or not to accept it, and if it is accepted, which channel to assign it to.

We measure the performance of a channel-assignment algorithm for such a system by its carried traffic function $T(r)$, which is the average carried traffic as a function of the average offered traffic $r$. We shall see that there is a function $T^{*}(r)$, which can be computed by linear programming, such that for any channel-assignment algorithm, $T(r) \leq T^{*}(r)$. On the other hand, we shall show that asymptotically as the number of available channels becomes large, there exist channel-assignment algorithms whose performance approaches $T^{*}(r)$ arbitrarily closely. Thus $T^{*}(r)$ can fairly be called the carried traffic function for the given system. The largest value of $r$ such that $T^{*}(r)=r$ is significant; it is the largest traffic intensity such that (asymptotically) all call requests can be honored. This value we call the capacity of the cellular MCS.

Here is a formal description of a cellular MCS. Let $S=$ $\left\{v_{1}, v_{2}, \ldots, v_{N}\right\}$ be the set of "cells," and let $\mathcal{S}=\left\{V_{1}, V_{2}, \ldots, V_{M}\right\}$ be a collection of subsets of $S$, called "maximal reuse sets." These sets represent the maximal sets of cells within which it is permissible to use the same channel simultaneously. We also define the incidence matrix $A=\left(a_{i j}\right)$ as

$$
a_{i j}=\left\{\begin{array}{ll}
1 & \text { if } v_{i} \in V_{j} \\
0 & \text { if } v_{i} \notin V_{j}
\end{array} .\right.
$$

The size of the $j$ th maximal reuse set is then $N_{j}$, where

$$
N_{j}=\left|V_{j}\right|=\sum_{i=1}^{N} a_{i j} .
$$

If we denote the number of active channels in cell $i$ by $m_{i}$, then the expected value of the carried traffic is $E\left(\sum_{i=1}^{N} m_{i}\right)$. We measure the performance of a given channel-assignment algorithm by its carried-traffic function $T(r)=(1 / n) E\left(m_{1}+\cdots+m_{N}\right)$. The function $T(r)$ represents the expected number of accepted calls per available channel. Our main result is the following:

Theorem 1. Suppose $T^{*}(r)$ is the value of the following linear program:

$$
r \sum_{i=1}^{N} p_{i} y_{i}+y_{N+1}=\text { minimum, subject to }
$$

Kumar N. Sivarajan

IBM T. J. Watson Research Center

P. O. Box 704

Yorktown Heights, NY 10598

$$
\begin{aligned}
& 0 \leq y_{i} \leq 1 \quad i=1,2, \ldots, N \\
& \sum_{i=1}^{N} y_{i} a_{i j}+y_{N+1} \geq N_{j} \quad j=1,2, \ldots, M .
\end{aligned}
$$

Then for any channel-assignment algorithm, $T(r) \leq T^{*}(r)$. Conversely, for any fixed $r$, there exists a channel-assignment algorithm whose asymptotic carried traffic function is arbitrarily close to $T^{*}(r)$.

The next theorem summarizes the most important properties of the function $T^{*}(r)$

Theorem 2. The function $T^{*}(r)$ has the following properties.

(a) $T^{*}(r)$ is nondecreasing, continuous, piecewise linear, and convex $\cap$.

(b) $T^{*}(r)=r$ for all $r \leq r_{0}$, where $r_{0}$ is the value of the following linear program:

$$
r_{0}=\max \left\{r: x \geq 0, x u_{M}^{T}=1, A x^{T} \geq r p^{T}\right\},
$$

where $u_{M}$ is the all-ones vector of dimension $M$.

(c) $T^{*}(r)=\max _{j} N_{j}$ for all $r \geq r_{1}$, where $r_{1}$ is the value of the following linear program:

$$
r_{1}=\min \left\{r: x \geq 0, x u_{M_{1}}^{T}=1, A_{1} x^{T} \leq r p^{T}\right\},
$$

where $V_{1}, V_{2}, \ldots, V_{M_{1}}$ are the maximal reuse sets of largest cardinality, and $A_{1}$ is the matrix consisting of the first $M_{1}$ columns of A.

Example. Consider the 19-cell example of Figure 1, with uniform traffic, i.e., $p=(1 / 19, \ldots, 1 / 19)$. In an attempt to model a real system in which signals propagate isotropically and attenuate according to an inverse fourth-power law, we define the reuse sets as follows: $V$ is a reuse set if and only if $\sum_{v \in V-\{u\}} d(u, v)^{-4} \leq 3 / 8$ for all $u \in V$, where $d(u, v)$ is the Euclidean distance between the center of cell $u$ and the center of cell $v$, and the distance between the centers of adjacent cells is 1 . It turns out that there are exactly 187 maximal reuse sets of this kind, and the carried-traffic function function is as shown in Figure 1. Note that the breakpoint $A$ shows that $r_{0}=247 / 49=5.04$, so the capacity of this system is 5.04 calls per channel. The breakpoint $F$ shows that $r_{1}=19$, so that the system is capable of carrying at most 7 calls per channel, which can be achieved only at the expense of a minimum "blocking" probability of $1-7 / 19=12 / 19=0.632$
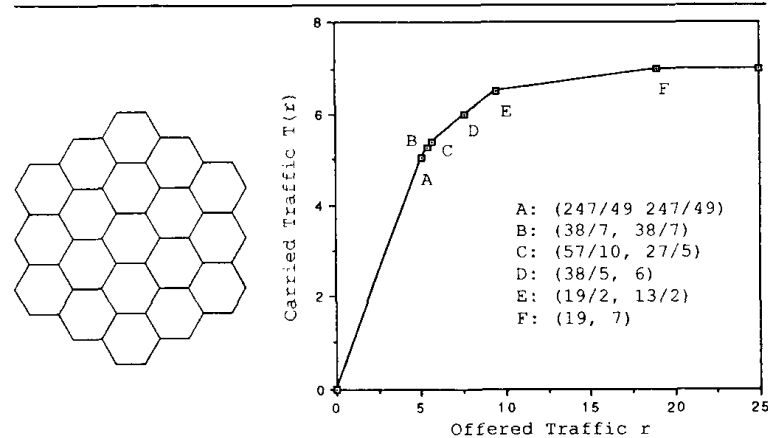

Figure 1. A 19-cell system, and its $T^{*}(r)$ curve.

\section{Acknowledgements}

This work was partially supported by a grant from GTE, AFOSR grant 88-0247, and a grant from Pacific Bell. 